\title{
Alterations in the gut microbiota of patients with silica-induced pulmonary fibrosis
}

\author{
Yao Zhou ${ }^{1 \dagger}$, Lv Chen ${ }^{1 \dagger}$, Gaofeng Sun ${ }^{2}$, Ying Li ${ }^{3}$ and Ruixue Huang ${ }^{1 *}$
}

\begin{abstract}
Silicosis resulting from silica exposure is a global occupational disease characterized by severe pathological changes in progressive pulmonary fibrosis. Previous evidence has indicated that dysbiosis of the gut microbiota occurs after environmental dust exposure and is associated with certain diseases. The aims of this study are to elucidate the compositional and functional characteristics of the gut microbiota in early-stage silicosis and to understand their influence on pulmonary fibrosis. We investigated the gut microbial composition of fecal samples from 18 patients and 21 healthy subjects using $16 \mathrm{~S}$ rRNA gene sequencing technology. Compared with the healthy subjects, reductions in the levels of Firmicutes and Actinobacteria were noted in patients with silicosis and progressive pulmonary fibrosis, as well as lower levels of Devosia, Clostridiales, AlloprevotellaandRikenellaceae_RC9.

Lachnospiraceae and Lachnoclostridium levels were increased in patients with silicosis. GOC and KEGG analyses were used to predict that certain bacteria taxa play critical roles in the development of pulmonary fibrosis, including posttranslational modification, amino acid transport and metabolism, nucleotide transport and metabolism, and ribosomal structure and biogenesis. KEGG analysis showed that certain taxa participate in various roles including cancer, endocrine metabolism, immune system, signaling molecules and interaction, and transcription. Collectively, in this pilot study, microbiota changes have been represented in the gut of patients with silicosis. Although this change in gut microbiota have been represented, caution is needed when interpreting the findings since this is observational finding, not necessarily causative. More studies should be performed in the expanding population to be verified and more studies underlying biological mechanisms for better understanding the relationship between gut microbiota and development of pulmonary fibrosis in patients with silicosis.
\end{abstract}

Keywords: Silicosis, Gut microbiota

\section{Introduction}

Silicosis, an occupational progressive fibrotic pulmonary disorder induced by inhaling free silicon dioxide or silica, is a public health concern that occurs commonly in many chemical and physical industries such as petroleum industries and iron and steel enterprises worldwide [1-3]. Silicosis is most common in low- and middle-income countries. In China, for instance, 600,000 cases of silicosis have been recorded over the past three decades, and this number continues to gradually rise

\footnotetext{
* Correspondence: huangruixue@csu.edu.cn

†Yao Zhou and Lv Chen contributed equally to this work.

${ }^{1}$ Department of Occupational and Environmental Health, Xiangya School of

Public Health, Changsha 410078, China

Full list of author information is available at the end of the article
}

each year, according to a report in 2016 [4]. In India, the rate of silicosis is estimated to range from 3.5 to $54.6 \%$ in various industries [5]. However, these values may be underestimated due to poor surveillance within developing countries. This public health concern is also prevalent in developed countries. The UK reported over 600,000 workers exposed to silica between 1990 to 1993, and silicosis-related deaths occur every year [6]. Workers in the US are also at a high risk of silica exposure, and silicosis-related deaths ranged from 3600 to 7300 annually between 1987 and 1996 [6]. Numerous studies have identified that inhalation of free silicon dioxide or silica is the cause of silicosis, and that the main clinical pathological feature is progressive pulmonary fibrosis [7]. The underlying mechanisms may involve

(c) The Author(s). 2019 Open Access This article is distributed under the terms of the Creative Commons Attribution 4.0 International License (http://creativecommons.org/licenses/by/4.0/), which permits unrestricted use, distribution, and 
silica-induced cytotoxicity of macrophages, activation of leukocytes to produce reactive oxygen free radicals, and damage to alveolar epithelial cells to induce fibroblast proliferation [8-10]. However, the exact mechanism of progressive pulmonary fibrosis in silicosis remains unclear.

Recent research on the lung-gut axis has revealed that the gut microbiota plays a critical role in maintaining pulmonary health, becoming a new frontier in the study of pulmonary-related diseases [11-13]. The gut microbiota, defined as the diverse microbial community that colonizes the host's gastrointestinal (GI) tract [14], has been evaluated in various pulmonary disorders. Barcik et al. investigated the diversity of the gut microbiota in fecal samples obtained from healthy individuals and asthma patients; the results indicated that some bacterial strains are partially responsible for enhancing histamine secretion, with Escherichia coli and Morganella morganii significantly elevated in the gut microbiome of patients with asthma [15]. Dickson reported that "Even slight differences in the abundance of healthy gut bacteria have been implicated in diverse systemic diseases" [16]. For instance, butyrate, which is produced in the gut and provides primary energy for lung epithelial cells, which induce the development of regulatory $\mathrm{T}$ cells to dampen the immune response, is decreased in the guts of patients with respiratory disease [17]. The study by Tamburini and Clemente demonstrated that neonatal gut microbiota induced pulmonary immunity, helping prevent pneumonia, in neonates $[18,19]$. Therefore, the gut microbiota has a role in maintaining lung health, and changes in gut microbiota diversity may lead to lung disease. Generally, many factors, including diet, lifestyle behaviors and environment, can modify the composition of the gut microbiota [20]. For example, alcohol is commonly considered to be a modulator of gut microbiota composition [21]. Dust in the home is an environmental factor that shapes the gut microbiota in infants infected with representative classes of bacteria(Actinobacteria, Bacilli, Clostridia and Gammaproteobacteria), showing a significant increase in dust-stool pairs compared with randomly permuted pairs [22]. Therefore, it was hypothesized that occupational exposure to silica modifies the composition of gut microbiota, which in turn is associated with progressive pulmonary fibrosis in patients with silicosis. To test this hypothesis, we performed microarray analysis of the fecal microbiota from patients with silicosis and from healthy individuals using $16 \mathrm{~S}$ ribosomal RNA gene sequencing. Our results showed that patients with silicosis have a gut microbiota community profile distinct from that of healthy individuals. This knowledge may be useful for early diagnosis of silicosis and for developing a method to inhibit the pulmonary fibrosis.

\section{Material and methods}

\section{Study design and recruitment of subjects}

Eighteen male patients with silicosis were recruited from the Occupational Disease Prevention and Treatment Hospital from October 2017 to January 2018. The baseline general socioeconomic status was recorded including age, urban or agrarian resident, smoking status, ethnicity as shown in Table 1 . Twenty-one sex-, age- and body mass index (BMI)-matched healthy individuals were also recruited as the control group. All patients with silicosis were diagnosed by at least three occupational disease physicians according to Chinese silicosis diagnostic standards (GBZ70-2015), based mainly on occupational exposure history, clinical symptoms, lung function investigation, X-ray examinations and silica levels reported in the workplace [23]. The inclusion criteria for the patients were males aged 18-70 years with symptoms of pathological fibrosis and their first diagnosis of silicosis without any history of clinical treatment as well as not use antibiotics and probiotics over the past sixmonths. The exclusion criteria for all enrolled participants were as follows: alcohol addiction; smoking history; complications of hypertension, diabetes, obesity, tuberculosis, asthma, chronic obstructive pulmonary disease or other related severe pulmonary diseases (e.g., lung cancer); $\mathrm{BMI}>27 \mathrm{~kg} / \mathrm{m} 2$; or use of antibiotics, probiotics, Chinese traditional herbal medicines or hormonal medications over the past 3 months. Twenty one healthy individuals underwent physical and liver biochemistry examinations, routine blood and urine tests, as well as serological tests to exclude any individuals with human immunodeficiency viral or hepatitis $\mathrm{C}$ or $\mathrm{B}$ viral infections and those with the abovementioned exclusion criteria.The silicosis process is divided into four phases clinically:Iphase,IIphase, IIIphase, and IVphase. The higher the phase, the more severity of silicosis is. Staging determination of silicosis requires assessment of the pneumonogram presentation including diffusionly distribute dots, small nodes and silicotic nodule. Considering silicosis is a development disorder and most of patients with IIIphase and IVphaseare treated with medication as well as accompanied with complications, we recruited Iphase and IIphase patients in this study.However, all silicosis patients, no matter how high the levels are, the presentation is lung fibrosis in common.

Above inclusion and exclusion criteria were created to make sure that the patients and control populations are similar in all terms except silica exposure. All enrolled subjects were informed of the nature of the study and were required to sign an informed consent form. All participants had the right to drop out of the study at any time without providing any explanation, and their information was kept confidential. The study has been approved by the Ethics Committee of Xiangya School of 
Table 1 Characteristics of enrolled subjects

\begin{tabular}{|c|c|c|c|c|c|c|}
\hline \multirow[t]{2}{*}{ Values } & & \multicolumn{2}{|c|}{ Patients with silicosis (A group) } & \multicolumn{2}{|c|}{ Healthy controls (B group) } & \multirow[t]{2}{*}{$P$ value } \\
\hline & & $N=18$ & $\%$ & $N=21$ & $\%$ & \\
\hline Age(year) & & $56.7 \pm 6.98$ & & $50.2 \pm 8.32$ & & 0.35 \\
\hline \multicolumn{7}{|l|}{ Education level } \\
\hline Elementary school(n) & & 3 & & 4 & & \\
\hline Junior high school(n) & & 15 & & 14 & & \\
\hline University or beyond(n) & & 0 & & 3 & & 0.43 \\
\hline \multicolumn{7}{|l|}{ Resident } \\
\hline Urban & & 2 & & 15 & & \\
\hline Agrarian & & 16 & & 6 & & 0.06 \\
\hline \multicolumn{7}{|l|}{ Smoking } \\
\hline Smoker & & 3 & & 10 & & \\
\hline Non-smoking & & 10 & & 5 & & \\
\hline Ex-smoker & & 5 & & 6 & & 0.17 \\
\hline \multicolumn{7}{|l|}{ Ethnicity } \\
\hline Han nationality & & 16 & & 18 & & \\
\hline Minority & & 2 & & 3 & & 0.45 \\
\hline \multicolumn{7}{|l|}{ Marital status } \\
\hline Married(n) & & 14 & & 17 & & \\
\hline Single/divorced/separated(n) & & 4 & & 4 & & 0.28 \\
\hline \multicolumn{7}{|l|}{ Occupation } \\
\hline Machine operator(n) & & 5 & & & & \\
\hline Mining operator(n) & & 11 & & & & \\
\hline Metalworking(n) & & 2 & & & & \\
\hline Administrator(n) & & & & 10 & & \\
\hline Office worker(n) & & & & 11 & & 0.04 \\
\hline $\mathrm{BMI}\left(\mathrm{kg} / \mathrm{m}^{2}\right)$ & & $21.64 \pm 7.5$ & & $22.7 \pm 5.43$ & & 0.59 \\
\hline \multirow[t]{2}{*}{ Silicosis phase } & 1 & 14 & 70 & & & \\
\hline & $\|$ & 6 & 30 & & & \\
\hline Symptoms lasting time before diagnosis(Month) & & $11 \pm 5$ & & & & \\
\hline \multirow[t]{2}{*}{ Silica level } & $<0.05 \mathrm{mg} / \mathrm{m}^{3}$ & 3 & 15 & & & \\
\hline & $>0.05 \mathrm{mg} / \mathrm{m}^{3}$ & 17 & 85 & & & \\
\hline \multirow[t]{3}{*}{ Fung function } & $\mathrm{FVC}(L)$ & $2.44 \pm 0.58$ & & $4.05 \pm 0.89$ & & $0.04^{*}$ \\
\hline & FEV1(L) & $2.45 \pm 0.64$ & & $3.37 \pm 1.01$ & & $0.04^{*}$ \\
\hline & $\mathrm{MW}(\mathrm{L})$ & $86.2 \pm 6.47$ & & $121.3 \pm 7.26$ & & $0.04^{*}$ \\
\hline \multirow[t]{2}{*}{$X$-ray result } & Slight lung fibrosis & 8 & 40 & & & \\
\hline & Severe lung fibrosis & 12 & 60 & & & \\
\hline Platelet $(10 \times 9 / L)$ & & $232.5 \pm 51.9$ & & $234.6 \pm 67.4$ & & 0.79 \\
\hline $\operatorname{WBC}(10 \times 9 / L)$ & & $5.53 \pm 2.9$ & & $5.79 \pm 3.04$ & & 0.58 \\
\hline $\mathrm{Hb}(\mathrm{g} / \mathrm{L})$ & & $115.5 \pm 49.4$ & & $139.2 \pm 39.5$ & & $<0.01$ \\
\hline
\end{tabular}

WBC white blood cell, $\mathrm{Hb}$ hemoglobin, FVC forced vital capacity, FEV1 forced expiratory volume in the first second. MVV: maximueP value for age and BMI was evaluated by pearson chi-square test

$* p<0.05$

Public Health. The study was conducted adhered to standard biosecurity and institutional safety procedures.

Fecal sample collection and DNA extraction

All participants in the study were required to undergo an overnight fast of at least $8 \mathrm{~h}$ in duration, after which blood $(5 \mathrm{~mL})$ samples were collected and stored at $4{ }^{\circ} \mathrm{C}$ for further routine blood and serological tests. All fecal samples were collected using disposable sterile forceps in the morning after at least an 8-h fast. If participants were unable to provide a fecal sample at the hospital, they had the option to collect the fecal 
sample at home and send it to the hospital on ice within $2 \mathrm{~h}$. All fecal samples were immediately divided into aliquots and stored at $-80^{\circ} \mathrm{C}$ for further DNA extraction.

Bacterial DNA extraction from the fecal samples was conducted at BioMARKER TECHNOLOGIES (Company, Beijing, China) using a Qiagen mini kit (Qiagen, Hilden, Germany). DNA was quantified using the Qubit 2.0 Fluorometer (Invitrogen, Carlsbad, CA, USA), and molecular size was estimated by agarose gel electrophoresis. All fecal microbial DNA was diluted to $10 \mathrm{ng} / \mu \mathrm{L}$ for microbial analysis.

The 16S rRNA gene was amplified by PCR using the following universal primers targeting the $\mathrm{V} 3-\mathrm{V} 4$ region of 16S rRNA: 338F 5'-ACTCCTACGGGAGG CAGCA-3' and 806R 5' -GGACTACHVGGGTWTCTA AT-3'. Each reaction contained $4 \mu \mathrm{L} 5 \times$ Fast Pfu.

Buffer (TransGen Biotech, Beijing, China), $2 \mu \mathrm{L} 2.5$ mM dNTPs, $0.8 \mu \mathrm{L}$ each primer $(5 \mu \mathrm{M}), 0.4 \mu \mathrm{L}$ Fast Pfu Polymerase and $10 \mathrm{ng}$ template DNA. Four PCR replicates were run per sample in a thermocycler (Eppendorf Mastercycler under the following parameters: $95^{\circ} \mathrm{C}$ for $2 \mathrm{~min}$, followed by 25 cycles of $95^{\circ} \mathrm{C}$ for $30 \mathrm{~s}, 55^{\circ} \mathrm{C}$ for $30 \mathrm{~s}$ and $72{ }^{\circ} \mathrm{C}$ for $45 \mathrm{~s}$, and a final extension at $72^{\circ} \mathrm{C}$ for $10 \mathrm{~min}$. Reactions from the same sample were pooled, purified by agarose gel separation and band extraction (Axygen Biosciences, Union City, CA, USA), and quantified using a fluorometric kit (Quant-iTPicoGreen, Invitrogen).

\section{Species identification and classification}

Bacteria16S:Silva (release 128, http://www.arb-silva.de) was used for species identification and analysis. Phylogenetic tree analysis was conducted using the neighbor-joining method, and multiple comparisons were performed using PyNAST (version 1.2.2, http://biocore.github.io/pynast/) and ClustalW2 [10] (http:// www.ebi.ac.uk/Tools/msa/clustalw2/).

\section{Diversity analysis \\ Alpha diversity analysis}

Alpha diversity is an index representing the diversity within a specific region or ecological system. Microbiota richness is commonly assessed using the Chao $\mathrm{l}$ and Ace estimators of species richness, and common measures of biodiversity are the Shannon-Wiener index and Simpson's index. Higher diversity is represented by a higher Shannon-Wiener index and a lower Simpson's index. The statistical analyses were conducted using Mothur software, version 1.30 (http://www.mothur.org/).

\section{Beta diversity analysis}

Beta diversity is an index used to analyze spatiotemporal variations in species composition. The species diversity was assessed using the binary Jaccard, Bray-Curtis and weighted UniFrac measures. Principal component analysis, principal coordinate analysis and relative analyses of environmental factors and samples (RDA/CCA) were performed using $\mathrm{R}$ software. Using principal component and principal coordinate analyses, the main parameters were identified and the differences among individuals or groups investigated. However, the former principal component analysis is the preferred method because it is based on the original species composition matrix, while the latter principal coordinate analysis is based on the distance matrix obtained from species composition arithmetic.

\section{Cluster analysis}

The unweighted pair group method with arithmetic mean was used for cluster analysis. The detailed procedure was as follows: the two smallest operational taxonomic units (OTUs) were clustered to form a new OTU, and then the mean distance between this new OTU and the other OTUs was calculated to find the next two smallest OTUs to cluster, continuing this process until all OTUs were clustered to form a phylogenetic tree.

\section{Heat map analysis}

The logarithm of each OTU was calculated, and the largest number of the top 80 was selected to perform heat map analysis using $\mathrm{R}$ software. In the heat map, each color lump represents a category richness of a fecal sample. The similarities in the microbiota community among fecal samples could be determined by the heat map.Mothur software (version 1.36.1)was used to obtain OTU table either in the study which was employed following the standard operating procedures from the website.

\section{LEfSe and Metastats analyses}

LEfSe analysis (http://huttenhower.sph.harvard.edu/ lefse/), a tool used to identify biomarkers, was used to estimate the effect of each species richness value on the variance. The aim of this method is to identify those species with the most significant variance in species richness between two groups. Metastats analysis (http:// metastats.cbcb.umd.edu/) was performed to identify the potential species causing the variance between the two groups using t-tests, with $p$-values $<0.05$, and Q-values $<$ 0.05 were considered significant.

\section{Function and statistical analysis}

COG (Clusters of Orthologous Groups) and KEGG(Kyoto Encyclopedia of Genes and Genomes) analyses were performed to predict the functions of the bacteria involved in the lung fibrosis in patients with silicosis compared with healthy subjects. COG database provide 
a tool to identification of orthologous genes which may be involved in various housekeeping functions especially those for transport and metabolism [24]. KEGG pathway analysis, is a predictive functional analysis using PICRUSt (phylogenetic investigation of communities by reconstruction of unobserved states) [25]. R software was used for all statistical analyses and construction of graphs. Values are presented as means and standard deviations, and $\mathrm{t}$-tests were used to assess the significance of differences in microbial taxa, clinical parameters and diversity indices. $p<0.05$ was considered to indicate significance.

\section{Results}

\section{Characteristics of the study participants}

In the study, 18 patients with silicosis and 20 healthy individuals were enrolled after applying the inclusion and exclusion criteria. No significant differences were found between the two groups in terms of age, BMI and platelet and white blood cell counts. However, hemoglobin levels were found to be significantly different between the two groups $(p<0.01)$ (Table 1$)$.

\section{Gut microbial diversity in patients with silicosis compared} with healthy subjects

To explore whether patients with silicosis exhibit differences in their gut microbiota compared with healthy subjects, the DNA from fecal samples was sequenced and assessed using IM_TORNEDO [26]. Figure 1a shows the number of OTUs detected in each sample from the patients and controls. The results suggested a different OUT number in patients with silicosis compared with healthy subjects (Fig. 1b). The Shannon-Wiener diversity index, used to evaluate species richness and evenness, was lower in the patients with silicosis compared with the control group (Fig. 1c), and Fig. 1d shows at phylum level, relative abundance(\%) of proteobacteria,

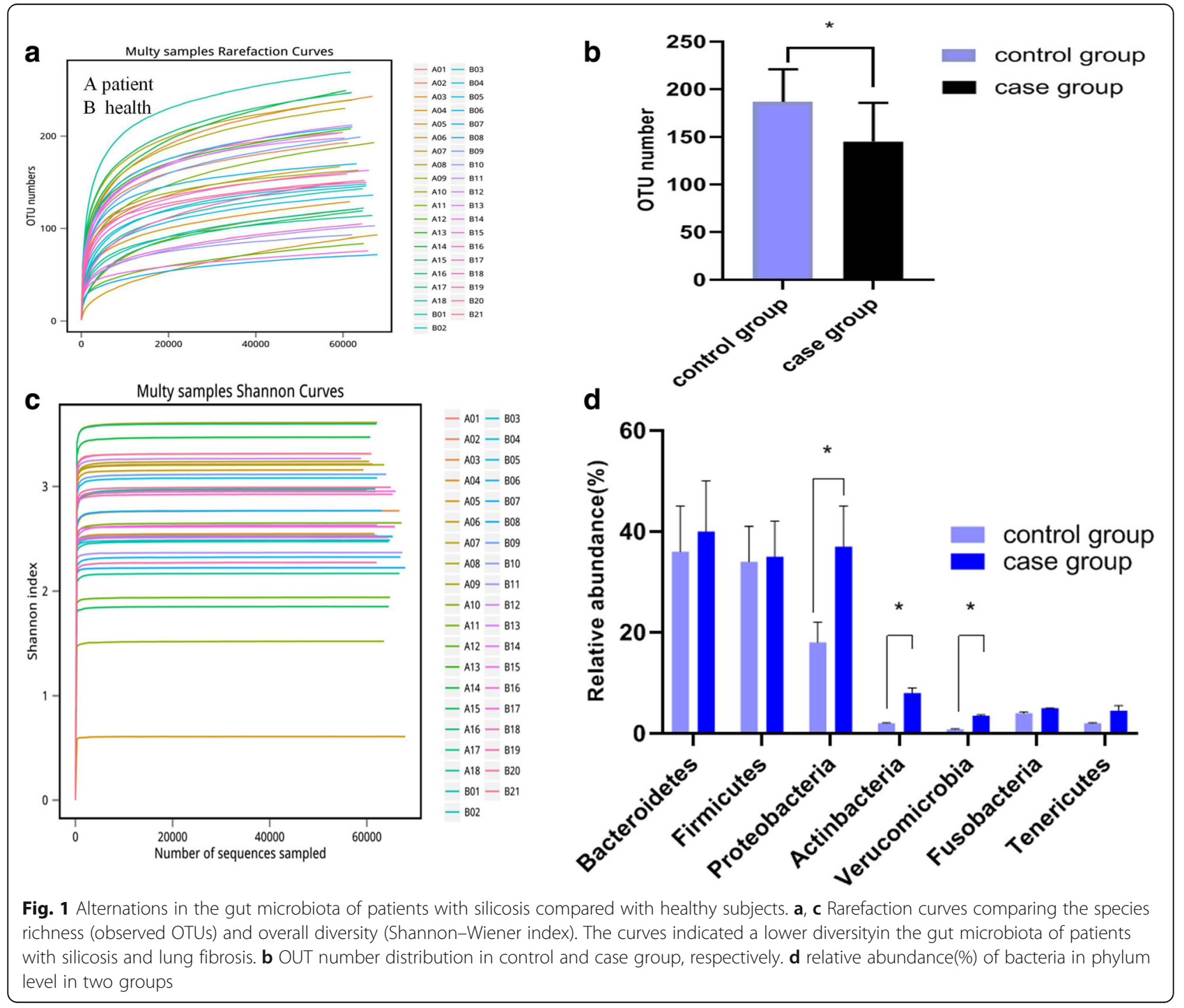


actinbacteria and verucomicrobia were increased in case group compared to control group.

To explore spatiotemporal changes in species, beta diversity analysis, including cluster trees, distance boxplots, and heat map analysis, was performed based on the Bray-Curtis distance matrix. Significant differences in gut microbiota diversity were observed between healthy subjects and patients with silicosis (Fig. 2).

\section{The abundances of certain bacteria are associated with silicosis}

To explore the specific bacterial taxa associated with silicosis, the LEfSe and Metastats methods were used to compare the fecal microbiota composition between the two groups. The cladogram in Fig. 3a shows the gut microbiota community structures and the differences in in the predominant bacterial taxa between the two groups. Briefly, the LEfSe analysis revealed seven discriminating features indicating different bacterial abundances in fecal samples between the patients and controls (LDA score $>4, p<0.05$, Fig. 3b).

The fecal abundances of Bacteroides and Escherichia-Shigella were decreased in the gut of the patients, whereas those of Megamonas, Lachnospiraceae, Lachnoclostridium, and Parabacteroides were increased (Fig. 3c-d). These taxa may be potential patheogenesis for silicosis. Changes in the gut microbiota composition of patients with silicosis were also explored using the Metastats method. Table 2 shows the differential taxon abundances between patients and healthy subjects at the phylum level. At the phylum level, the abundancesof Actinobacteria, Acidobacteria, Gemmatimonadetes, Saccharibacteria, Fusobacteria, Aminicenantes and Verrucomicrobia were lower in patients with silicosis than in a

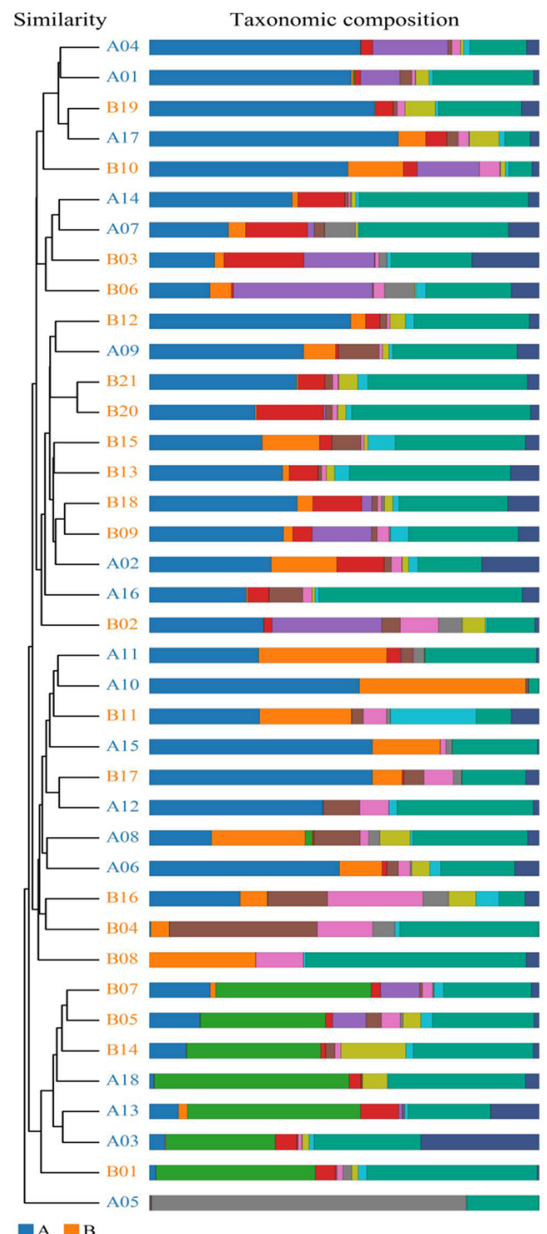

b
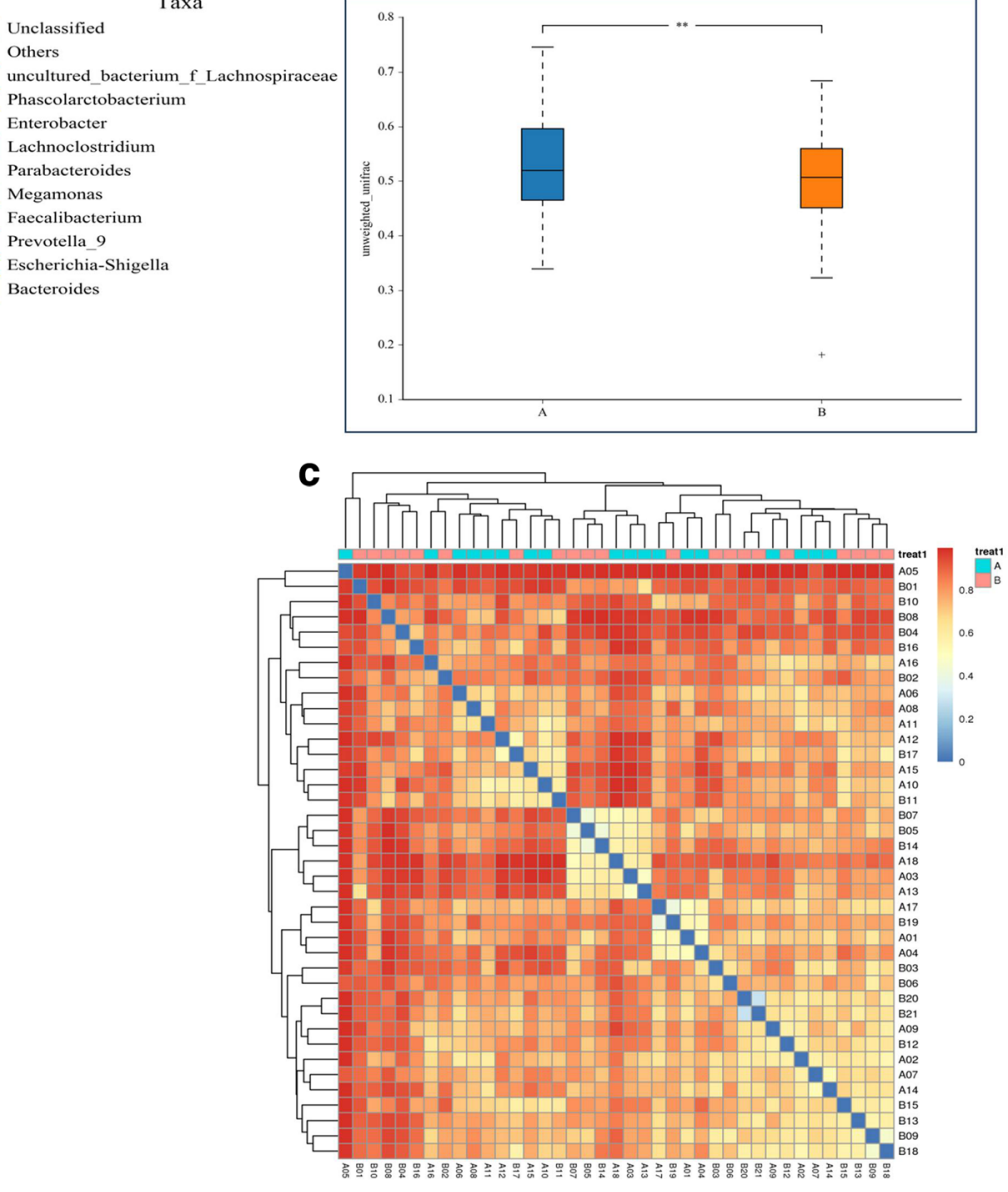

Fig. 2 Beta diversity analysis of gut microbiota among patients with silicosis and healthy subjects. a A cluster tree showing significant gut microbiotadiversity in patients with silicosis. $\mathbf{b}$ Boxplots depicting differences in gut microbiota diversity between the two groups. Box parameters, the "-" symbol represents the median value, and the upper and lower ranges of the box represent the 75th and 25th percentiles, respectively. c A heat map depicting the relationship between the two groups. The blue color represents the range of $0-0.4$ and the red color the range of $0.4-1.0$ 


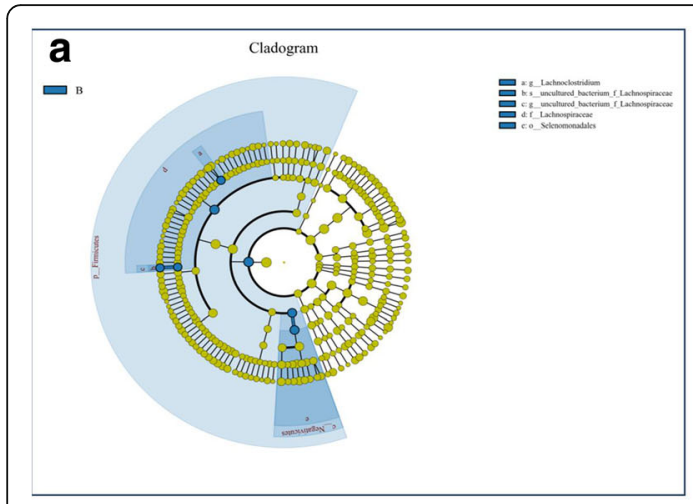

C

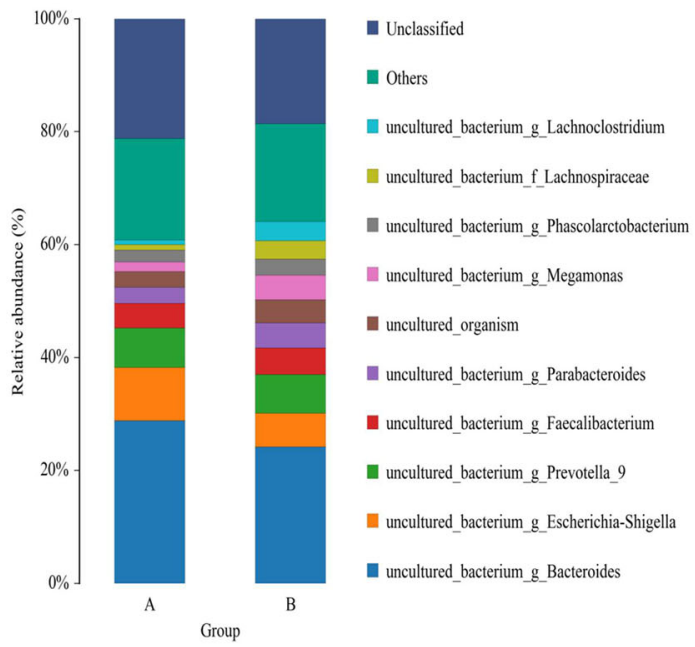

b

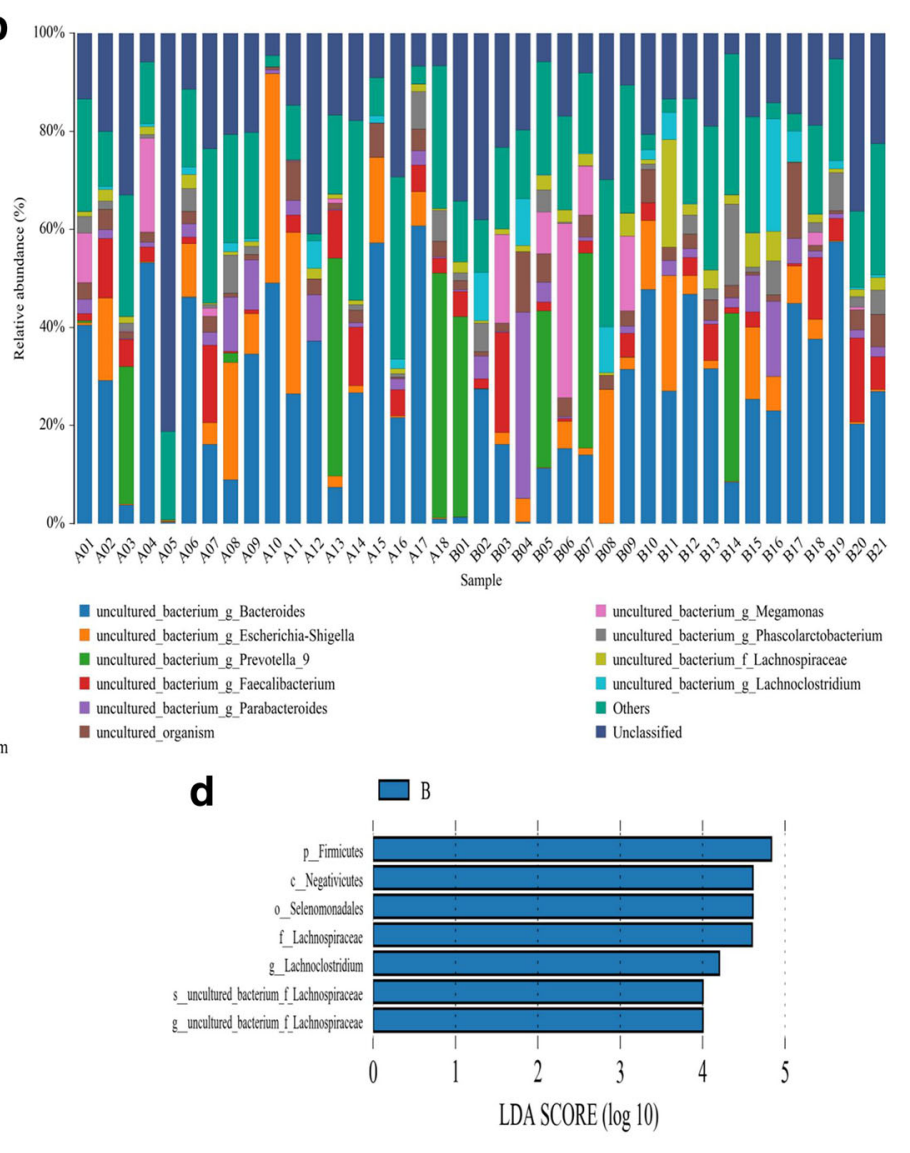

Fig. 3 The abundances of certain bacteria are associated with silicosis. a A cladogram showing the gut microbiota community structures as well as the different predominant bacteria between the two groups. $\mathbf{b}$ Seven discriminating features obtained by LEfSe analysis indicating different bacterial abundances between the groups. $\mathbf{c}$ The abundances of the fecal species Bacteroides and Escherichia-Shigella were decreased in the guts of patients, whereas those ofMegamonas,Lachnospiraceae,Lachnoclostridium,and Parabacteroides were increased at genuslevel (c-d). d The features indicating different abundances in the fecal samples between the patients with silicosis and healthy controls (LDA score $>4, p<0.05$, d)

healthy subjects $(\mathrm{p}<0.05)$, whereas those of Proteobacteria, Synergistetes, Lentisphaerae, Tenericutes and Cyanobacteria were higher in patients with silicosis than in healthy subjects $(\mathrm{p}<0.05)$. At the species level, the abundances of Subdoligranulum, Blautia, prokaryote, Nitrosomonadaceae, Bifidobacterium and Pseudomonas_aeruginosa were decreased in the fecal samples of the patients, whereas those of Megamonas,Dialiste and uncultured_bacterium_g_Ruminiclostridum_6 were significantly increased compared with the healthy controls (Fig. 4a). These data demonstrate different abundances of certain bacteria in the gut microbiota ofpatients with silicosis compared with healthy subjects, and the gut dysbiosis in patients with silicosis may be associated with the aberrant fecal microbiota composition.

\section{Function prediction}

COG and KEGG analyses were performed to predict the functions of the bacteria involved in the lung fibrosis in patients with silicosis compared with healthy subjects (Fig. 4b-c). According to the COG predictions, the bacteria represented in the patients with silicosis are mainly associated with the following biological processes: cell wall/membrane/envelope biogenesis, carbohydrate transport and metabolism, transcription, inorganic ion transport, and coenzyme transport and metabolism. According to the KEGG predictions, the bacteria represented in the patients with silicosis are mainly associated with the following biological processes: xenobiotic biodegradation and metabolism, cell motility, translation, nucleotide metabolism, metabolism of terpenoids and polyketides, signal transduction and membrane transport.

\section{Discussion}

To the best of our knowledge, although the association between the gut microbiota and pulmonary disease has drawn much attention, and studies have been conducted 
Table 2 Differential taxa abundances between two groups at phylum level

\begin{tabular}{|c|c|c|c|c|c|c|c|c|}
\hline & \multicolumn{3}{|c|}{ Healthy subjects(A) } & \multicolumn{3}{|c|}{ Patients with silicosis(B) } & \multirow[t]{2}{*}{$P$ value } & \multirow{2}{*}{$\begin{array}{l}\mathrm{Q} \\
\text { value }\end{array}$} \\
\hline & Mean & Variance & SE & Mean & Variance & SE & & \\
\hline \multicolumn{9}{|l|}{ Phylum } \\
\hline Firmicutes & 0.329 & 0.0274 & 0.039 & 0.472 & 0.0170 & 0.0284 & 0.004 & 0.04 \\
\hline Actinobacteria & 0.009 & 0.0004 & 0.004 & 0.001 & 0 & 0 & 0.0008 & 0.04 \\
\hline \multicolumn{9}{|l|}{ Family } \\
\hline Veillonellaceae & 0.0298 & 0.00248 & 0.0117 & 0.102 & 0.0168 & 0.0283 & 0.027 & 0.0753 \\
\hline Lachnospiraceae & 0.136 & 0.014 & 0.0279 & 0.227 & 0.0172 & 0.0286 & 0.034 & 0.0887 \\
\hline Enterobacteriaceae & 0.169 & 0.0447 & 0.0498 & 0.0777 & 0.00641 & 0.0175 & 0.0849 & 0.19 \\
\hline Enterococcaceae & 0.0102 & 0.00171 & 0.0097 & 0.00005 & 0 & 0 & 0.169 & 0.297 \\
\hline Bacteroidaceae & 0.324 & 0.0431 & 0.0489 & 0.272 & 0.0318 & 0.0389 & 0.42 & 0.559 \\
\hline Clostridiales_vadinBB60_group & 0.0003 & 0.00000 & 0.0003 & 0 & 0 & 0 & 0.000999 & 0.00405 \\
\hline Leptotrichiaceae & 0 & 0 & 0 & 0.00004 & 0 & 0 & 0.00099 & 0.00405 \\
\hline Succinivibrionaceae & 0 & 0 & 0 & 0.00019 & 0 & 0.00019 & 0.00099 & 0.00405 \\
\hline Bradyrhizobiaceae & 0.0002 & 0 & 0.0001 & 0 & 0 & 0 & 0.016 & 0.0518 \\
\hline Streptococcaceae & 0.0043 & 0.00006 & 0.0019 & 0.00067 & 0 & 0.00022 & 0.02 & 0.0622 \\
\hline \multicolumn{9}{|l|}{ genus } \\
\hline Devosia & 0.0000147 & 0 & 0 & 0 & 0 & 0 & 0 & 0.00037 \\
\hline Ruminofilibacter & 0.000014 & 0 & 0 & 0 & 0 & 0 & 0 & 0.00041 \\
\hline Bosea & 0.000013 & 0 & 0 & 0 & 0 & 0 & 0 & 0.00044 \\
\hline Jatrophihabitans & 0.0000131 & 0 & 0 & 0 & 0 & 0 & 0 & 0.00044 \\
\hline uncultured_bacterium_f_Blastocatellaceae_ & 0.0000145 & 0 & 0 & 0 & 0 & 0 & 0 & 0.00148 \\
\hline uncultured_bacterium_f_JTB255_marine_benthic_group & 0 & 0 & 0 & 0 & 0 & 0 & 0 & 0.00216 \\
\hline Anaeroglobus & 0.0000134 & 0 & 0 & 0 & 0 & 0 & 0.00013 & 0.00317 \\
\hline
\end{tabular}

on this association in certain lung diseases, including asthma, chronic obstructive pulmonary diseaseand viral lung infection [27, 28], the knowledge gap concerning the relationship between the gut microbiota and pulmonary fibrosis remains.

Emerging evidence has demonstrated that the human gut microbiota can confer either health benefits or a susceptibility to disorders.It has been reported that exposure to silica was associated with occurrence of systemic sclerosis [29]. Furthermore, Andreasson K et al. analyzed the prevalence of intestinal dysbiosis in systemic sclerosis patients and indicated that a majority $(75.5 \%)$ of the patients exhibited intestinal dysbiosis, which was more severe in patients with pulmonary fibrosis [30]. Based on these previous studies, it is suggested that patients with silica-induced pulmonary fibrosis may exhibit dysbiosis of gut microbiota. Using 16S rRNA sequencing techniques, in this study, we have found possibilities that patients with silicosis have gut microbial changes. Our study revealed compositional differences in the gut microbiota of patients with silicosis compared with healthy subjects. At the phylum level,Firmicutes and Actinobacteriaabundances were lower in patients with silicosis $(p<0.05)$. The COG and KEGG prediction analyses showed the altered gut microbiota in patients with silicosis to be associated with numerous biological functions at the cell and systemic levels. Although this change in gut microbiota have been represented, caution is needed when interpreting the findings since this is observational finding, not necessarily causative.

The majority of the human gut/fecal microbiota is comprised of either the Firmicutes or Actinobacteria [31, 32]. Disruption of the balance between the Firmicutes and Actinobacteriamay increase the risk of many disorders, such as insulin resistance and obesity, as outlined in previous reports [33,34]. In our study, we consistently observed a decreasing trend in levels of the Firmicutes and Actinobacteriain patients with silicosis with progressive pulmonary fibrosis. Although animal studies have linked gut dysbiosis with an increased risk of pulmonary fibrosis later in life [35], our study is the first to show a relationship between silica-induced progressive pulmonary fibrosis and altered gut microbiota diversity.

At the genus level, some studies have indicated that the species abundance level may be associated with maintaining body health. A decreased abundance of Devosiais associated with irritable bowel syndrome in 


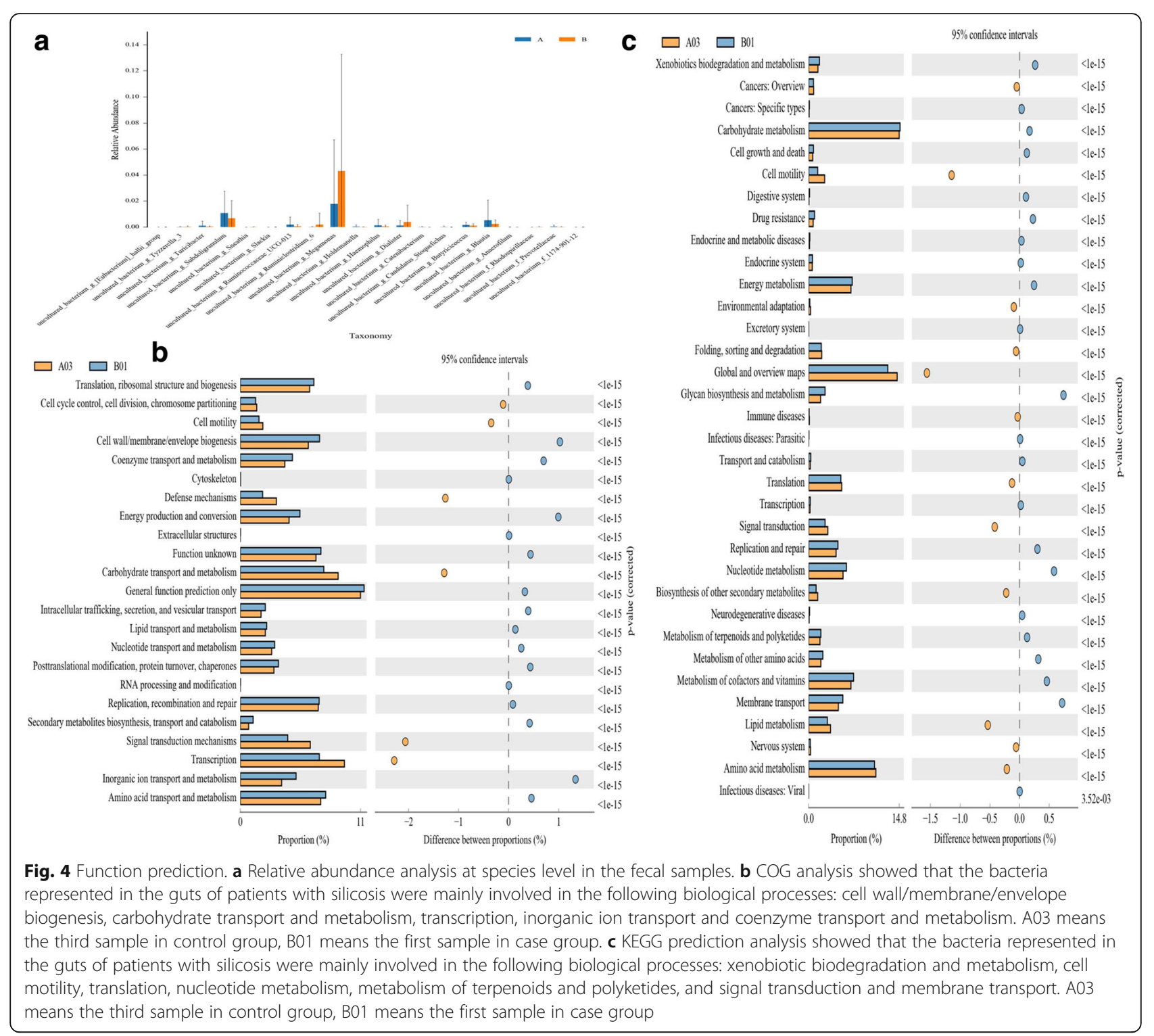

humans [36]. Kelly et al. found an association between the risk of cardiovascular disease and Alloprevotella abundance, which was decreased in patients in the Bogalusa Heart study [37]. Our study is consistent with previous studies that showed lower levels of Devosia, Clostridiales, Alloprevotella and Rikenellaceae_RC9 in fecal samples from patients with silicosis compared with healthy subjects. Decreased levels of these fecal bacteria may lead to a poor immune ability, resulting in failure to prevent progressive pulmonary fibrosis. However, it should be mentioned that in the LDA analysis in our study, no exciting and interesting differences showed between two group although the statistical results showed $p$ value was under 0.05 . This phenomenon might due to the small samples enrolled in this study, further investigations on larger participants' samples should be considered to conduct the LDA analysis based on more larger population.

Lachnospiraceae and Lachnoclostridium levels were found to be increased in some diseases. A greater abundance of Lachnospiraceae was found in HIV-infected patients [38]. McCall et al. also found altered Lachnospiraceae levels in patients with Chagas disease [39]. Lachnoclostridium abundance was increased in patients who had been exposed to the antibiotic cefprozil [40]. Our study is consistent with these results, in that Lachnospiraceae and Lachnoclostridium levels were increased in patients with silicosis. The increased levels of Lachnospiraceae and Lachnoclostridium may contribute to gut disease susceptibility or inhibit metabolic pathways associated with progressive pulmonary fibrosis, eventually leading to silicosis. The detailed mechanisms 
by which the increased or decreased abundance levels of certain bacteria initiate the development of pulmonary fibrosis in patients with silicosis require further investigation.

A key advantage of this study is the prediction of bacterial functions using GOC and KEGG analyses. However, further mechanism studies should be performed to verify the roles of these potential bacteria in the development of pulmonary fibrosis.

Some limitations of the study should be mentioned. First, the samples sizes were small, which may affect explaining the conclusions. Large size of samples should be considered to further vilify the difference between case group and normal group. Second, some differences are relatively small rather than significant, this lead to the cautions while interpret these results. Third, dietary component for the patients which may have altered as a result of symptoms which in turn have altered the microbiota was not considered in this study. Forth, study in two groups according to our criteria would be possible affected by some cofounders, such as both two groups may be exposedto other pollutants such as particulate matter, location of housing, different childhood and companionships(e.g. originatingmore or less from agrarian culture), concomitant diseases and medications. For instance, ambient $\mathrm{PM}_{2.5}$ causes gut dysbiosis and may subsequently contribute to developing glucose metabolism abnormalities [41]. Geographical location, such as urban and rural areas, is associated with alterations of gut microbiota [42]. Therefore, for further study, we should formulate more critical inclusion and exclusion criteria to make the interpretation of results more reliable. In conclusion, we report biological community composition occurred differently in control group and silicosis patients.Moreover, the levels of Firmicutes and Actinobacteria were reduced in patients with silicosis. This may give us a deeper understanding on the possible role of gut microbiota in the pathogenesis of silica-induced pulmonary fibrosis.

\section{Funding}

This study was supported by the National Natural Science Foundation of China (No. U1803124).

\section{Availability of data and materials}

N/A

\section{Authors' contributions}

RXH contributed study concept and critical design, YZ, GFS, LC, YL conducted the cell experiments. LC acquired, analyzed and interpreted data. LC, facilitated quality assurance of data. YZ, GFS, LC fulfill the initial manuscript and RXH critically reviewed and revised the final manuscript. All authors were involved in the current study, contributed to this study. All authors read and approved the final manuscript.
Consent for publication

This work is published under the standard license to publish agreement.

Competing interests

The authors declare that they have no competing interests.

\section{Publisher's Note}

Springer Nature remains neutral with regard to jurisdictional claims in published maps and institutional affiliations.

\section{Author details}

${ }^{1}$ Department of Occupational and Environmental Health, Xiangya School of Public Health, Changsha 410078, China. ${ }^{2}$ Department of Chronic and Non-communicable Diseases Control, City Center for Disease Control and Prevention, Urumqi 830026, China. ${ }^{3}$ Hunan Prevention and Treatment Center For Occupational Diseases, Changsha, China.

Received: 2 November 2018 Accepted: 24 February 2019

Published online: 04 March 2019

\section{References}

1. Knight D, Ehrlich R, Fielding K, Jeffery H, Grant A, Churchyard G. Trends in silicosis prevalence and the healthy worker effect among gold miners in South Africa: a prevalence study with follow up of employment status. BMC Public Health. 2015;15:1258.

2. Huang $R, Y u T, L i Y, H u$ J. Upregulated has-miR-4516 as a potential biomarker for early diagnosis of dust-induced pulmonary fibrosis in patients with pneumoconiosis. Toxicol Res. 2018;7:415-22.

3. Huang R, Zhou P. Double-edged effects of noncoding RNAs in responses to environmental genotoxic insults: perspectives with regards to moleculeecology network. Environ Pollut. 2019;247:64-71.

4. Ning H, Zhou Y, Zhou Z, Cheng S, Huang R, Ning H, Huang R. Challenges to improving occupational health in China. Occup Environ Med. 2017;74:924-5.

5. Sharma N, Kundu D, Dhaked S, Das A. Silicosis and silicotuberculosis in India. Bull World Health Organ. 2016;94:777-8.

6. Leung CC, Yu IT, Chen W. Silicosis. Lancet. 2012;379:2008-18.

7. Bhattacharya S, Dey A, Pal A, Kar S, Saha S. Silicosis in the form of progressive massive fibrosis: a diagnostic challenge. Indian J Occup Environ Med. 2016;20:114-7.

8. Qin J, Ning H, Zhou Y, Hu Y, Yang L, Huang R. LncRNA MIR31HG overexpression serves as poor prognostic biomarker and promotes cells proliferation in lung adenocarcinoma. Biomed Pharmacother. 2018;99:363-8.

9. Mo LJ, Song M, Huang QH, Guan H, Liu XD, Xie DF, Huang B, Huang RX, Zhou PK. Exosome-packaged miR-1246 contributes to bystander DNA damage by targeting LIG4. Br J Cancer. 2018;119:492-502.

10. Zhou PK, Huang RX. Targeting of the respiratory chain by toxicants: beyond the toxicities to mitochondrial morphology. Toxicol Res (Camb). 2018;7: 1008-11.

11. Wang L, Hao K, Yang T, Wang C. Role of the lung microbiome in the pathogenesis of chronic obstructive pulmonary disease. Chin Med J. 2017; 130:2107-11.

12. Shukla SD, Budden KF, Neal R, Hansbro PM. Microbiome effects on immunity, health and disease in the lung. Clin Transl Immunol. 2017;6:e133.

13. Huang R, Wang K, Hu J. Effect of probiotics on depression: a systematic review and meta-analysis of randomized controlled trials. Nutrients. 2016;8. https://doi.org/10.3390/nu8080483.

14. Holmes E, Li JV, Athanasiou T, Ashrafian H, Nicholson JK. Understanding the role of gut microbiome-host metabolic signal disruption in health and disease. Trends Microbiol. 2011:19:349-59.

15. Barcik W, Pugin B, Westermann P, Perez NR, Ferstl R, Wawrzyniak M, Smolinska S, Jutel M, Hessel EM, Michalovich D, Akdis CA, Frei R, O'Mahony L. Histamine-secreting microbes are increased in the gut of adult asthma patients. J Allergy Clin Immunol. 2016;138:1491-4 e7.

16. Dickson RP. The microbiome and critical illness. Lancet Respir Med. 2016;4: 59-72.

17. Shimizu K, Ogura H, Hamasaki T, Goto M, Tasaki O, Asahara T, Nomoto K, Morotomi M, Matsushima A, Kuwagata $Y$, Sugimoto $H$. Altered gut flora are associated with septic complications and death in critically ill patients with systemic inflammatory response syndrome. Dig Dis Sci. 2011:56:1171-7.

18. Gray J, Oehrle K, Worthen G, Alenghat T, Whitsett J, Deshmukh H. Intestinal commensal bacteria mediate lung mucosal immunity and promote 
resistance of newborn mice to infection. Sci Transl Med. 2017;9. https://doi. org/10.1126/scitranslmed.aaf9412

19. Tamburini S, Clemente JC. Gut microbiota: neonatal gut microbiota induces lung immunity against pneumonia. Nat Rev Gastroenterol Hepatol. 2017;14: 263-4.

20. Liang D, Leung RK, Guan W, Au WW. Involvement of gut microbiome in human health and disease: brief overview, knowledge gaps and research opportunities. Gut Pathogens. 2018;10:3.

21. Chen Y, Yang F, Lu H, Wang B, Chen Y, Lei D, Wang Y, Zhu B, Li L. Characterization of fecal microbial communities in patients with liver cirrhosis. Hepatology. 2011;54:562-72.

22. Konya T, Koster B, Maughan H, Escobar M, Azad MB, Guttman DS, Sears MR, Becker AB, Brook JR, Takaro TK, Kozyrskyj AL, Scott JA, Investigators CS. Associations between bacterial communities of house dust and infant gut. Environ Res. 2014:131:25-30.

23. Tang SJ, Zhu YS. Several issues on diagnosis and treatment of silicosis and tuberculosis. Zhonghua Jie He He Hu Xi Za Zhi. 2011;34:568-70.

24. Galperin MY, Makarova KS, Wolf Yl, Koonin EV. Expanded microbial genome coverage and improved protein family annotation in the COG database. Nucleic Acids Res. 2015;43:D261-9.

25. Guzzardi MA, Ali LA, D'Aurizio R, Rizzo F, Saggese P, Sanguinetti E, Weisz A, Pellegrini M, lozzo P. Fetal cardiac growth is associated with in utero gut colonization. Nutr Metab Cardiovasc Dis. 2019:29(2):170-176.

26. Jeraldo P, Kalari K, Chen X, Bhavsar J, Mangalam A, White B, Nelson H, Kocher JP, Chia N. IM-TORNADO: a tool for comparison of 165 reads from paired-end libraries. PLoS One. 2014;9:e114804.

27. Engel M, Endesfelder D, Schloter-Hai B, Kublik S, Granitsiotis MS, Boschetto P, Stendardo M, Barta I, Dome B, Deleuze JF, Boland A, Muller-Quernheim J, Prasse A, Welte T, Hohlfeld J, Subramanian D, Parr D, Gut IG, Greulich T, Koczulla AR, Nowinski A, Gorecka D, Singh D, Gupta S, Brightling CE, Hoffmann $H$, Frankenberger M, Hofer TP, Burggraf D, Heiss-Neumann M, Ziegler-Heitbrock L, Schloter M, Castell WZ. Influence of lung CT changes in chronic obstructive pulmonary disease (COPD) on the human lung microbiome. PLoS One. 2017:12:e0180859.

28. Groves HT, Cuthbertson L, James P, Moffatt MF, Cox MJ, Tregoning JS. Respiratory disease following viral lung infection alters the murine Gut microbiota. Front Immunol. 2018;9:182.

29. Alaya Z, Kalboussi H, Osman W, Naouar N, Zeglaoui H, Bouajina E. Silicaassociated systemic sclerosis occurring after an occupational exposure to arc welding. Pan Afr Med J. 2016;25:70.

30. Andreasson K, Alrawi Z, Persson A, Jonsson G, Marsal J. Intestinal dysbiosis is common in systemic sclerosis and associated with gastrointestinal and extraintestinal features of disease. Arthritis Res Ther. 2016;18:278.

31. Jami M, Ghanbari M, Kneifel W, Domig KJ. Phylogenetic diversity and biological activity of culturable Actinobacteria isolated from freshwater fish gut microbiota. Microbiol Res. 2015;175:6-15.

32. Berry $D$. The emerging view of Firmicutes as key fibre degraders in the human gut. Environ Microbiol. 2016;18:2081-3.

33. Riva A, Borgo F, Lassandro C, Verduci E, Morace G, Borghi E, Berry D. Pediatric obesity is associated with an altered gut microbiota and discordant shifts in Firmicutes populations. Environ Microbiol. 2017;19:95-105.

34. Greenhill C. Gut microbiota: Firmicutes and Bacteroidetes involved in insulin resistance by mediating levels of glucagon-like peptide 1. Nat Rev Endocrinol. 2015;11:254.

35. Ho KJ, Varga J. Early-life Gut Dysbiosis: a driver of later-life fibrosis? J Invest Dermatol. 2017;137:2253-5.

36. Ng SC, Lam EF, Lam TT, Chan Y, Law W, Tse PC, Kamm MA, Sung JJ, Chan FK, Wu JC. Effect of probiotic bacteria on the intestinal microbiota in irritable bowel syndrome. J Gastroenterol Hepatol. 2013;28:1624-31.

37. Kelly TN, Bazzano LA, Ajami NJ, He H, Zhao J, Petrosino JF, Correa A, He J. Gut microbiome associates with lifetime cardiovascular disease risk profile among Bogalusa heart study participants. Circ Res. 2016;119:956-64.

38. San-Juan-Vergara H, Zurek E, Ajami NJ, Mogollon C, Pena M, Portnoy I, Velez Jl, Cadena-Cruz C, Diaz-Olmos Y, Hurtado-Gomez L, Sanchez-Sit S, Hernandez D, Urruchurtu I, Di-Ruggiero P, Guardo-Garcia E, Torres N, VidalOrjuela O, Viasus D, Petrosino JF, Cervantes-Acosta G. A Lachnospiraceaedominated bacterial signature in the fecal microbiota of HIV-infected individuals from Colombia, South America. Sci Rep. 2018;8:4479.

39. McCall LI, Tripathi A, Vargas F, Knight R, Dorrestein PC, Siqueira-Neto JL. Experimental Chagas disease-induced perturbations of the fecal microbiome and metabolome. PLoS Negl Trop Dis. 2018;12:e0006344.
40. Raymond F, Ouameur AA, Deraspe M, Iqbal N, Gingras H, Dridi B, Leprohon P, Plante PL, Giroux R, Berube E, Frenette J, Boudreau DK, Simard JL, Chabot I, Domingo MC, Trottier S, Boissinot M, Huletsky A, Roy PH, Ouellette M, Bergeron MG, Corbeil J. The initial state of the human gut microbiome determines its reshaping by antibiotics. ISME J. 2016;10:707-20.

41. Wang W, Zhou J, Chen M, Huang X, Xie X, Li W, Cao Q, Kan H, Xu Y, Ying Z. Exposure to concentrated ambient PM2.5 alters the composition of gut microbiota in a murine model. Part Fibre Toxicol. 2018;15:17.

42. Angelakis E, Bachar D, Yasir M, Musso D, Djossou F, Gaborit B, Brah S, Diallo A, Ndombe GM, Mediannikov O, Robert C, Azhar El, Bibi F, Nsana NS, Parra HJ, Akiana J, Sokhna C, Davoust B, Dutour A, Raoult D. Treponema species enrich the gut microbiota of traditional rural populations but are absent from urban individuals. New Microbes New Infect. 2019;27:14-21.
Ready to submit your research? Choose BMC and benefit from:

- fast, convenient online submission

- thorough peer review by experienced researchers in your field

- rapid publication on acceptance

- support for research data, including large and complex data types

- gold Open Access which fosters wider collaboration and increased citations

- maximum visibility for your research: over $100 \mathrm{M}$ website views per year

At BMC, research is always in progress.

Learn more biomedcentral.com/submissions 